5-1-2014

\title{
Median Based Modified Ratio Estimators with Known Quartiles of an Auxiliary Variable
}

Jambulingam Subramani

PondicherryUniversity, Puducherry, India, drjsubramani@yahoo.co.in

G Prabavathy

Pondicherry University, Puducherry, India, praba.gopal.23@gmail.com

Follow this and additional works at: http://digitalcommons.wayne.edu/jmasm

Part of the Applied Statistics Commons, Social and Behavioral Sciences Commons, and the Statistical Theory Commons

\section{Recommended Citation}

Subramani, Jambulingam and Prabavathy, G (2014) "Median Based Modified Ratio Estimators with Known Quartiles of an Auxiliary Variable," Journal of Modern Applied Statistical Methods: Vol. 13 : Iss. 1, Article 15.

DOI: $10.22237 /$ jmasm/1398917640

Available at: http://digitalcommons.wayne.edu/jmasm/vol13/iss1/15

This Regular Article is brought to you for free and open access by the Open Access Journals at DigitalCommons@WayneState. It has been accepted for inclusion in Journal of Modern Applied Statistical Methods by an authorized editor of DigitalCommons@WayneState. 


\section{Median Based Modified Ratio Estimators with Known Quartiles of an Auxiliary Variable}

\section{Cover Page Footnote}

The authors thank the referees and the Editor for their comments and suggestions which have improved the presentation of the paper. The authors wish to record their gratitude and thanks to the University Grants Commission, New Delhi for having given the financial assistance to carry out this research work through the UGC-Major Research Project. 


\section{Median Based Modified Ratio Estimators with Known Quartiles of an Auxiliary Variable}

\author{
Jambulingam Subramani \\ Pondicherry University \\ Puducherry, India
}

\author{
G. Prabavathy \\ Pondicherry University \\ Puducherry, India
}

New median based modified ratio estimators for estimating a finite population mean using quartiles and functions of an auxiliary variable are proposed. The bias and mean squared error of the proposed estimators are obtained and the mean squared error of the proposed estimators are compared with the usual simple random sampling without replacement (SRSWOR) sample mean, ratio estimator, a few existing modified ratio estimators, the linear regression estimator and median based ratio estimator for certain natural populations. A numerical study shows that the proposed estimators perform better than existing estimators; in addition, it is shown that the proposed median based modified ratio estimators outperform the ratio and modified ratio estimators as well as the linear regression estimator.

Keywords: $\quad$ Bias, inter-quartile range, linear regression estimator, mean squared error, natural population, simple random sampling

\section{Introduction}

Consider a finite population $U=\left\{U_{1}, U_{2}, \ldots, U_{N}\right\}$ of $N$ distinct and identifiable units. Let $Y$ be a study variable with value $Y_{i}$ measured on $U_{i}, i=1,2,3, \ldots, N$ giving a vector $Y=\left\{Y_{1}, Y_{2}, \ldots, Y_{N}\right\}$. The goal is to estimate the population mean, $\bar{Y}=\frac{1}{N} \sum_{i=1}^{N} Y_{i}$, with some desirable properties on the basis of a random sample of size $n$ selected from the population $U$. The simplest estimator of population mean is the sample mean, obtained by using simple random sampling without

Dr. Subramani is Associate Professor and Head, Department of Statistics. Email him at: drjsubramani@yahoo.co.in. G. Prabavathy is a Project Fellow in the UGC-MRP and a Doctoral Student in the Department of Statistics. Email her at:

praba.gopal.23@gmail.com. 


\section{SUBRAMANI \& PRABAVATHY}

replacement (SRSWOR), when there is no information on the auxiliary variable available. Let $X$ be an auxiliary variable that is positively correlated with the study variable $Y$ : Sometimes the information on auxiliary variable $X$, positively correlated with $Y$, may be utilized to obtain a more efficient estimator of the population mean (for further details on ratio estimators see Cochran, 1977 and Murthy, 1967.) When the population parameters of an auxiliary variable $X$, such as, population mean, coefficient of variation, coefficient of kurtosis, coefficient of skewness and median are known, ratio, product and linear regression estimators (and their modifications) have been proposed in the literature - many of which perform better than the SRSWOR sample mean for estimating the population mean of a study variable.

Subramani (2013a) proposed a median based ratio estimator by using the median of a study variable as auxiliary information, and it has been shown that this median based ratio estimator outperforms the usual SRSWOR sample mean, ratio estimator, modified ratio estimator and linear regression estimator. Based on Subramani's (2013a) median based ratio estimator, some new median based modified ratio estimators with known quartiles of the auxiliary variable are proposed.

The first quartile, also called lower quartile, is denoted by $Q_{1}$; the third quartile, also called the upper quartile, is denoted by $Q_{3}$. The lower quartile is a point where $25 \%$ of the observations are less than $Q_{1}$ and $75 \%$ are above $Q_{1}$. The upper quartile is a point where $75 \%$ observations are less than $Q_{3}$ and $25 \%$ are above $Q_{3}$. Quartiles are unaffected by extreme values unlike the population mean, variance, correlation coefficient, etc.

The inter-quartile range used as a measure of spread in a data set. The interquartile range of a distribution is the difference between the upper and lower quartiles. The formula for computing the inter-quartile range is

$$
Q_{r}=Q_{3}-Q_{1} \text {. }
$$

The semi-quartile range of a distribution is half the difference between the upper and lower quartiles, or half the inter-quartile range. The formula for computing the semi-quartile range is

$$
Q_{d}=\frac{Q_{3}-Q_{1}}{2}
$$




\section{MEDIAN BASED MODIFIED RATIO ESTIMATORS}

Another measure, the quartile average, noted by $Q_{a}$, was suggested by Subramani and Kumarapandiyan (2012a) and is defined as

$$
Q_{a}=\frac{Q_{3}+Q_{1}}{2}
$$

The notations and formulae used in this article are:

$N$ : Population size

$n$ : Sample size

$Y$ : Study variable

$M$ : Median of the study variable

$X$ : Auxiliary variable

$Q_{i}: \quad i^{\text {th }}$ Quartile of auxiliary variable, $i=1,3$

$\rho: \quad$ Correlation coefficient between $X$ and $Y$

$\bar{X}, \bar{Y}: \quad$ Population means

$\bar{x}, \bar{y}: \quad$ Sample means

$\bar{M}: \quad$ Average of sample medians of $Y$

$m$ : Sample median of $Y$

$\beta$ : $\quad$ Regression coefficient of $Y$ on $X$

$B($.$) : Bias of the estimator$

$V($.$) : Variance of the estimator$

$M S E():$.$\quad Mean squared error of the estimator$

$\operatorname{PRE}(e, p)=\frac{\operatorname{MSE}(e)}{\operatorname{MSE}(p)} * 100 \quad \begin{aligned} & \text { Percent relative efficiency of the } \\ & \text { proposed estimator } p \text { with respect to the }\end{aligned}$

: $\quad$ existing estimator $e$

The formulae for computing various measures including the variance and the covariance of the SRSWOR sample mean and sample median are: 


\section{SUBRAMANI \& PRABAVATHY}

$$
\begin{aligned}
& V(\bar{y})=\frac{1}{N_{C_{n}}} \sum_{i=1}^{N_{C_{n}}}\left(\bar{y}_{i}-\bar{Y}\right)^{2}=\frac{1-f}{n} S_{y}^{2}, V(\bar{x})=\frac{1}{N_{C_{n}}} \sum_{i=1}^{N_{C_{n}}}\left(\bar{x}_{i}-\bar{X}\right)^{2}=\frac{1-f}{n} S_{x}^{2}, \\
& \operatorname{MSE}(m)=V(m)=\frac{1}{N_{C_{n}}} \sum_{i=1}^{N_{C_{n}}}\left(m_{i}-M\right)^{2}, \\
& \operatorname{Cov}(\bar{y}, \bar{x})=\frac{1}{N_{C_{n}}} \sum_{i=1}^{N_{C_{n}}}\left(\bar{x}_{i}-\bar{X}\right)\left(\bar{y}_{i}-\bar{Y}\right)=\frac{1-f}{n} \frac{1}{N-1} \sum_{i=1}^{N}\left(X_{i}-\bar{X}\right)\left(Y_{i}-\bar{Y}\right), \\
& \operatorname{Cov}(\bar{y}, m)=\frac{1}{N_{C_{n}}} \sum_{i=1}^{N_{C_{n}}}\left(m_{i}-M\right)\left(\bar{y}_{i}-\bar{Y}\right), \\
& C_{x x}^{\prime}=\frac{V(\bar{x})}{\bar{X}^{2}}, C_{m m}^{\prime}=\frac{V(m)}{M^{2}}, C_{y m}^{\prime}=\frac{\operatorname{Cov}(\bar{y}, m)}{M \bar{Y}}, C_{y x}^{\prime}=\frac{\operatorname{Cov}(\bar{y}, \bar{x})}{\bar{X} \bar{Y}}, \\
& \text { where } f=\frac{n}{N} ; S_{y}^{2}=\frac{1}{N-1} \sum_{i=1}^{N}\left(Y_{i}-\bar{Y}\right)^{2}, S_{x}^{2}=\frac{1}{N-1} \sum_{i=1}^{N}\left(X_{i}-\bar{X}\right)^{2} .
\end{aligned}
$$

In the case of SRSWOR, the sample mean, $\bar{y}$, is used to estimate the population mean, $\bar{Y}$. That is, the estimator of $\bar{Y}=\hat{\bar{Y}}_{r}=\bar{y}$ with variance

$$
V\left(\hat{\bar{Y}}_{r}\right)=\frac{1-f}{n} S_{y}^{2}
$$

The classical ratio estimator for estimating the population mean $\bar{Y}$ of a study variable $Y$ is defined as $\hat{\bar{Y}}_{R}=\frac{\bar{y}}{\bar{x}} \bar{X}=\hat{R} \bar{X}$. The bias and mean squared error of $\hat{\bar{Y}}_{R}$ are:

$$
B\left(\hat{\bar{Y}}_{R}\right)=\bar{Y}\left\{C_{x x}^{\prime}-C_{y x}^{\prime}\right\}
$$

and

$$
\operatorname{MSE}\left(\hat{\bar{Y}}_{R}\right)=V(\bar{y})+R^{2} V(\bar{x})-2 R \operatorname{Cov}(\bar{y}, \bar{x}) .
$$




\section{MEDIAN BASED MODIFIED RATIO ESTIMATORS}

The other commonly used estimator using the auxiliary variable $X$ is the linear regression estimator. The linear regression estimator and its variance with known regression coefficient are:

$$
\begin{gathered}
\hat{\bar{Y}}_{l r}=\bar{y}+\beta(\bar{X}-\bar{x}) \\
V\left(\hat{\bar{Y}}_{l r}\right)=V(\bar{y})\left(1-\rho^{2}\right) \text { where } \rho=\frac{\operatorname{Cov}(\bar{y}, \bar{x})}{\sqrt{V(\bar{x})^{*} V(\bar{y})}}
\end{gathered}
$$

Subramani \& Kumarapandiyan (2012a) suggested some modified ratio estimators using known quartiles and their functions of an auxiliary variable, these are:

$$
\begin{aligned}
& \hat{\bar{Y}}_{R M 1}=\bar{y}\left(\frac{\bar{X}+Q_{1}}{\bar{x}+Q_{1}}\right) \\
& \hat{\bar{Y}}_{R M 2}=\bar{y}\left(\frac{\bar{X}+Q_{3}}{\bar{x}+Q_{3}}\right) \\
& \hat{\bar{Y}}_{R M 3}=\bar{y}\left(\frac{\bar{X}+Q_{r}}{\bar{x}+Q_{r}}\right) \\
& \hat{\bar{Y}}_{R M 4}=\bar{y}\left(\frac{\bar{X}+Q_{d}}{\bar{x}+Q_{d}}\right) \\
& \hat{\bar{Y}}_{R M 5}=\bar{y}\left(\frac{\bar{X}+Q_{a}}{\bar{x}+Q_{a}}\right)
\end{aligned}
$$

The bias and the mean squared error of the modified ratio estimators in (9) to (13) are:

$$
B\left(\hat{\bar{Y}}_{R M i}\right)=\bar{Y}\left\{\theta_{i}^{2} C_{x x}^{\prime}-\theta_{i} C_{y x}^{\prime}\right\}
$$




\section{SUBRAMANI \& PRABAVATHY}

$$
\operatorname{MSE}\left(\hat{\bar{Y}}_{R M i}\right)=V(\bar{y})+R^{2} \theta_{i}^{2} V(\bar{x})-2 R \theta_{i} \operatorname{Cov}(\bar{y}, \bar{x})
$$

where $R=\frac{\bar{Y}}{\bar{X}}$ and

$$
\theta_{1}=\frac{\bar{X}}{\bar{X}+Q_{1}}, \theta_{2}=\frac{\bar{X}}{\bar{X}+Q_{3}}, \theta_{3}=\frac{\bar{X}}{\bar{X}+Q_{r}}, \theta_{4}=\frac{\bar{X}}{\bar{X}+Q_{d}}, \theta_{5}=\frac{\bar{X}}{\bar{X}+Q_{a}} ; i=1,2,3,4,5
$$

Recently Subramani (2013a) suggested a median based ratio estimator for estimating $\bar{Y}$ when the median of the study variable $Y$ is known. The estimator with its bias and mean squared error are:

$$
\begin{gathered}
\hat{\bar{Y}}_{M}=\frac{\bar{y}}{m} M \\
B\left(\hat{\bar{Y}}_{M}\right)=\bar{Y}\left\{C_{m m}^{\prime}-C_{y m}^{\prime}-\frac{\operatorname{Bias}(m)}{M}\right\} \\
\operatorname{MSE}\left(\hat{\bar{Y}}_{M}\right)=V(\bar{y})+R^{\prime 2} V(m)-2 R^{\prime} \operatorname{Cov}(\bar{y}, m) \text { where } R^{\prime}=\frac{\bar{Y}}{M} .
\end{gathered}
$$

For further details on modified ratio estimators with known population parameters of an auxiliary variable, such as coefficient of variation, skewness, kurtosis, correlation coefficient, quartiles and their linear combinations, readers are referred to Kadilar and Cingi (2004, 2006a, b, 2009) Koyuncu and Kadilar (2009), Singh and Kakran (1993), Singh and Tailor (2003, 2005), Singh (2003), Sisodia and Dwivedi (1981), Subramani (2013a, b), Subramani and Kumarapandiyan (2012a, b, c, 2013), Tailor and Sharma (2009), Tin (1965), and Yan and Tian (2010).

The median based ratio estimator proposed by Subramani (2013a) is extended and, as a result, some new median based modified ratio estimators $\hat{\bar{Y}}_{S P 1}$, $\hat{\bar{Y}}_{S P 2}, \hat{\bar{Y}}_{S P 3}, \hat{\bar{Y}}_{S P 4}$ and $\hat{\bar{Y}}_{S P 5}$ with known quartiles and their functions of auxiliary variables are proposed. 


\section{MEDIAN BASED MODIFIED RATIO ESTIMATORS}

\section{Proposed Median Based Modified Ratio Estimators}

The proposed median based modified ratio estimators for estimating a population mean $\bar{Y}$ based on Subramani's (2013a) ratio estimator are:

$$
\begin{aligned}
& \hat{\bar{Y}}_{S P 1}=\bar{y}\left(\frac{M+Q_{1}}{m+Q_{1}}\right) \\
& \hat{\bar{Y}}_{S P 2}=\bar{y}\left(\frac{M+Q_{3}}{m+Q_{3}}\right) \\
& \hat{\bar{Y}}_{S P 3}=\bar{y}\left(\frac{M+Q_{r}}{m+Q_{r}}\right) \\
& \hat{\bar{Y}}_{S P 4}=\bar{y}\left(\frac{M+Q_{d}}{m+Q_{d}}\right)
\end{aligned}
$$

and

$$
\hat{\bar{Y}}_{S P 5}=\bar{y}\left(\frac{M+Q_{a}}{m+Q_{a}}\right)
$$

To the first degree of approximation, the bias and mean squared error of $\hat{\bar{Y}}_{S P j}$ are derived as:

$$
\begin{gathered}
B\left(\hat{\bar{Y}}_{S P j}\right)=\bar{Y}\left\{\theta_{j}^{\prime 2} C_{m m}^{\prime}-\theta_{j}^{\prime} C_{y m}^{\prime}-\theta_{j}^{\prime} \frac{\operatorname{Bias}(m)}{M}\right\}, j=1,2,3,4,5, \\
\operatorname{MSE}\left(\hat{\bar{Y}}_{S P j}\right)=V(\bar{y})+R^{\prime 2} \theta_{j}^{\prime 2} V(m)-2 R^{\prime} \theta_{j}^{\prime} \operatorname{Cov}(\bar{y}, m), j=1,2,3,4,5
\end{gathered}
$$

where

$$
R^{\prime}=\frac{\bar{Y}}{M}, \theta_{1}^{\prime}=\frac{M}{M+Q_{1}}, \theta_{2}^{\prime}=\frac{M}{M+Q_{3}}, \theta_{3}^{\prime}=\frac{M}{M+Q_{r}}, \theta_{4}^{\prime}=\frac{M}{M+Q_{d}}, \theta_{5}^{\prime}=\frac{M}{M+Q_{a}} .
$$


See Appendix A for detailed derivation of the bias and the mean squared error of $\hat{\bar{Y}}_{S P j}$.

\section{Efficiency Comparisons}

\section{Comparison with SRSWOR Sample Mean}

The conditions (see Appendix B) for which the proposed estimators $\hat{\bar{Y}}_{S P j}, j=1,2,3,4,5$ are more efficient than the SRSWOR sample mean $\hat{\bar{Y}}_{r}$ were derived from expressions (25) and (4) and are:

$$
\operatorname{MSE}\left(\hat{\bar{Y}}_{S P j}\right) \leq V\left(\hat{\bar{Y}}_{r}\right) \text { if } 2 C_{y m}^{\prime} \geq \theta_{j}^{\prime} C_{m m}^{\prime} ; j=1,2,3,4,5
$$

\section{Comparison with Ratio Estimators}

The conditions (see Appendix B) for which the proposed estimators $\hat{\bar{Y}}_{S P j}, j=1,2,3,4,5$ are more efficient than the usual ratio estimator $\hat{\bar{Y}}_{R}$ were derived from expressions (25) and (6) and are:

$$
\operatorname{MSE}\left(\hat{\bar{Y}}_{S P j}\right) \leq \operatorname{MSE}\left(\hat{\bar{Y}}_{R}\right) \text { if } \theta_{j}^{\prime 2} C_{m m}^{\prime}-\theta_{i}^{2} C_{x x}^{\prime} \leq 2\left(\theta_{j}^{\prime} C_{y m}^{\prime}-\theta_{i} C_{y x}^{\prime}\right) ; i, j=1,2,3,4,5 .
$$

\section{Comparison with Modified Ratio Estimators}

From expressions (25) and (15), the conditions (see Appendix B) for which the proposed estimators $\hat{\bar{Y}}_{S P j}, j=1,2,3,4,5$ are more efficient than the existing modified ratio estimator $\hat{\bar{Y}}_{R M i}, i=1,2,3,4,5$ were derived and are:

$$
\operatorname{MSE}\left(\hat{\bar{Y}}_{S P j}\right) \leq \operatorname{MSE}\left(\hat{\bar{Y}}_{R M i}\right)
$$

if

$$
\operatorname{MSE}\left(\hat{\bar{Y}}_{S P j}\right) \leq \operatorname{MSE}\left(\hat{\bar{Y}}_{R M i}\right) \text { if } \theta_{j}^{\prime 2} C_{m m}^{\prime}-\theta_{i}^{2} C_{x x}^{\prime} \leq 2\left(\theta_{j}^{\prime} C_{y m}^{\prime}-\theta_{i} C_{y x}^{\prime}\right) ; i, j=1,2,3,4,5
$$




\section{MEDIAN BASED MODIFIED RATIO ESTIMATORS}

\section{Comparison with Linear Regression Estimator}

From expressions (25) and (8), the conditions (see Appendix B) for which the proposed estimators $\hat{\bar{Y}}_{S P j}, j=1,2,3,4,5$ are more efficient than the usual linear regression estimator $\hat{\bar{Y}}_{l r}$ were derived and are:

$$
\operatorname{MSE}\left(\hat{\bar{Y}}_{S P_{j}}\right) \leq V\left(\hat{\bar{Y}}_{l r}\right) \text { if } 2 \theta_{j}^{\prime} C_{y m}^{\prime}-\theta_{j}^{\prime 2} C_{m m}^{\prime} \geq \frac{\left[C_{y x}^{\prime}\right]^{2}}{C_{x x}^{\prime}} ; j=1,2 .
$$

\section{Comparison with Median Based Ratio Estimator}

From expressions (25) and (18), the conditions (see Appendix B) for which the proposed estimators $\hat{\bar{Y}}_{S P j}, j=1,2,3,4,5$ are more efficient than the existing modified ratio type estimator $\hat{\bar{Y}}_{M}$ were derived and are:

$$
\operatorname{MSE}\left(\hat{\bar{Y}}_{S P_{j}}\right) \leq \operatorname{MSE}\left(\hat{\bar{Y}}_{M}\right) \text { if } 2 C_{y m}^{\prime} \leq\left(\theta_{j}^{\prime}+1\right) C_{m m}^{\prime} ; j=1,2,3,4,5 .
$$

\section{Numerical Comparison}

The conditions for which the proposed median based modified ratio estimators performed better than the other usual estimators considered in this study have been obtained. In order to show that the proposed estimators perform better than the other estimators, numerical comparisons were made to determine the efficiencies of the proposed estimators. Two populations were used to assess the efficiencies of the proposed median based modified ratio estimators with that of the existing estimators. Populations 1 and 2 are from Singh and Chaudhary (1986, p. 177). The parameter values and constants computed for the populations are given in Table 1, the bias for the proposed and existing estimators computed for the two populations are given in Table 2 and the mean squared errors are given in Table 3. 


\section{SUBRAMANI \& PRABAVATHY}

Table 1. Parameter values and constants for 2 different populations

\begin{tabular}{|c|c|c|c|c|}
\hline \multirow{2}{*}{ Parameter } & \multicolumn{2}{|c|}{$n=3$} & \multicolumn{2}{|c|}{$n=5$} \\
\hline & Pop 1 & Pop 2 & Pop 1 & Pop 2 \\
\hline$N$ & 34.0000 & 34.0000 & 34.0000 & 34.0000 \\
\hline$n$ & 3.0000 & 3.0000 & 5.0000 & 5.0000 \\
\hline$N_{c n}$ & $5,984.0000$ & $5,984.0000$ & $278,256.0000$ & $278,256.0000$ \\
\hline $\bar{Y}$ & 856.4118 & 856.4118 & 856.4118 & 856.4118 \\
\hline $\bar{M}$ & 747.7223 & 747.7223 & 736.9811 & 736.9811 \\
\hline$M$ & 767.5000 & 767.5000 & 767.5000 & 767.5000 \\
\hline $\bar{X}$ & 208.8824 & 199.4412 & 208.8824 & 199.4412 \\
\hline$Q_{1}$ & 94.2500 & 99.2500 & 94.2500 & 99.2500 \\
\hline$Q_{3}$ & 254.7500 & 278.0000 & 254.7500 & 278.0000 \\
\hline$Q_{r}$ & 160.5000 & 178.7500 & 160.5000 & 178.7500 \\
\hline$Q_{d}$ & 80.2500 & 89.3750 & 80.2500 & 89.3750 \\
\hline$Q_{a}$ & 174.5000 & 188.6250 & 174.5000 & 188.6250 \\
\hline$R$ & 4.0999 & 4.2941 & 4.0999 & 4.2941 \\
\hline$R^{\prime}$ & 1.1158 & 1.1158 & 1.1158 & 1.1158 \\
\hline$\theta_{1}$ & 0.6891 & 0.6677 & 0.6891 & 0.6677 \\
\hline$\theta_{2}$ & 0.4505 & 0.4177 & 0.4505 & 0.4177 \\
\hline$\theta_{3}$ & 0.5655 & 0.5274 & 0.5655 & 0.5274 \\
\hline$\theta_{4}$ & 0.7224 & 0.6905 & 0.7224 & 0.6905 \\
\hline$\theta_{\mathrm{s}}$ & 0.5448 & 0.5139 & 0.5448 & 0.5139 \\
\hline$\theta_{1}^{\prime}$ & 0.8906 & 0.8855 & 0.8906 & 0.8855 \\
\hline$\theta_{2}^{\prime}$ & 0.7508 & 0.7341 & 0.7508 & 0.7341 \\
\hline$\theta_{3}^{\prime}$ & 0.8270 & 0.8111 & 0.8270 & 0.8111 \\
\hline$\theta_{4}^{\prime}$ & 0.9053 & 0.8957 & 0.9053 & 0.8957 \\
\hline$\theta_{s}^{\prime}$ & 0.8148 & 0.8027 & 0.8148 & 0.8027 \\
\hline $\operatorname{var}(\bar{y})$ & $163,356.4086$ & $163,356.4086$ & $91,690.3713$ & $91,690.3713$ \\
\hline $\operatorname{var}(\bar{x})$ & 6,884.4455 & $6,857.8555$ & $3,864.1726$ & $3,849.2480$ \\
\hline $\operatorname{var}(m)$ & $101,518.7738$ & $101,518.7738$ & $59,396.2836$ & $59,396.2836$ \\
\hline $\operatorname{cov}(\bar{y}, m)$ & $90,236.2939$ & $90,236.2939$ & $48,074.9542$ & $48,074.9542$ \\
\hline $\operatorname{cov}(\bar{y}, \bar{x})$ & $15,061.4011$ & $14,905.0488$ & $8,453.8187$ & $8,366.0597$ \\
\hline$\rho$ & 0.4491 & 0.4453 & 0.4491 & 0.4453 \\
\hline
\end{tabular}




\section{MEDIAN BASED MODIFIED RATIO ESTIMATORS}

Table 2. Bias of existing and proposed estimators

\begin{tabular}{|c|c|c|c|c|c|}
\hline \multirow{2}{*}{\multicolumn{2}{|c|}{ Estimators }} & \multicolumn{2}{|c|}{$n=3$} & \multicolumn{2}{|c|}{$n=5$} \\
\hline & & Pop 1 & Pop 2 & Pop 1 & Pop 2 \\
\hline \multirow{7}{*}{ Existing } & $\hat{\overline{Y_{R}}}$ & 63.0241 & 72.9186 & 35.3748 & 40.9285 \\
\hline & $\hat{\bar{Y}}_{R \mathrm{~N} 1}$ & 14.4774 & 15.9291 & 8.1261 & 8.9409 \\
\hline & $\hat{\bar{Y}}_{\text {RU }}$ & -5.0570 & -5.4535 & -2.8385 & -3.0610 \\
\hline & $\hat{\bar{Y}}_{\text {RU }}$ & 2.4369 & 1.6513 & 1.3678 & 0.9269 \\
\hline & $\hat{\bar{Y}}_{\text {Rus }}$ & 18.4357 & 18.8016 & 10.3478 & 10.5531 \\
\hline & $\hat{\bar{Y}}_{\text {Rus }}$ & 0.8276 & 0.5910 & 0.4645 & 0.3317 \\
\hline & $\hat{\bar{Y}}_{M}$ & 52.0924 & 52.0924 & 57.7705 & 57.7705 \\
\hline \multirow{5}{*}{ Proposed } & $\hat{\bar{Y}}_{s p 1}$ & 32.0179 & 31.1618 & 43.0405 & 42.3993 \\
\hline & $\hat{\bar{Y}}_{s p 2}$ & 11.4953 & 9.4306 & 27.2167 & 25.5531 \\
\hline & $\hat{\bar{Y}}_{S P 3}$ & 21.9708 & 19.6375 & 35.4268 & 33.6263 \\
\hline & $\hat{\bar{Y}}_{S p 4}$ & 34.5121 & 32.8700 & 44.9012 & 43.6773 \\
\hline & $\hat{\bar{Y}}_{s p s}$ & 20.1662 & 18.4422 & 34.0355 & 32.6983 \\
\hline
\end{tabular}

Table 3. Variance/mean squared error of existing and proposed estimators

\begin{tabular}{|c|c|c|c|c|c|}
\hline \multirow{2}{*}{\multicolumn{2}{|c|}{ Estimators }} & \multicolumn{2}{|c|}{$n=3$} & \multicolumn{2}{|c|}{$n=5$} \\
\hline & & Pop 1 & Pop 2 & Pop 1 & Pop 2 \\
\hline \multirow{9}{*}{ Existing } & $\hat{\bar{Y}}_{r}$ & 163356.4086 & 163356.4086 & 91690.3713 & 91690.3713 \\
\hline & $\hat{\bar{Y}}_{R}$ & 155577.8155 & 161802.8878 & 87324.3215 & 90818.3961 \\
\hline & $\hat{\bar{Y}}_{R M 1}$ & 133203.7861 & 134261.9210 & 74765.9957 & 75359.9173 \\
\hline & $\hat{\bar{Y}}_{R N 2}$ & 131205.2291 & 131950.5079 & 73644.2252 & 74062.5432 \\
\hline & $\hat{\bar{Y}}_{R U 3}$ & 130523.6191 & 131018.5135 & 73261.6440 & 73539.4239 \\
\hline & $\hat{\bar{Y}}_{R N A}$ & 134530.4901 & 135259.2456 & 75510.6618 & 75919.7060 \\
\hline & $\hat{\bar{Y}}_{R M S}$ & 130420.4186 & 130968.9816 & 73203.7186 & 73511.6221 \\
\hline & $\hat{\bar{Y}}$ & 130408.9222 & 130964.1249 & 73197.2660 & 73508.8959 \\
\hline & $\frac{\hat{\bar{Y}}}{w}$ & 88379.0666 & 88379.0666 & 58356.9234 & 58356.9234 \\
\hline \multirow{5}{*}{ Proposed } & $\hat{\bar{Y}}_{s p 1}$ & 84266.7092 & 84147.8927 & 54798.7634 & 54675.1252 \\
\hline & $\hat{\bar{Y}}_{s p 2}$ & 83413.6960 & 83642.1970 & 52826.6580 & 52784.4688 \\
\hline & $\hat{\bar{Y}}_{S P 3}$ & 83266.0122 & 83175.3231 & 53543.5010 & 53322.4123 \\
\hline & $\hat{\bar{Y}}_{S P 4}$ & 84643.7479 & 84390.4264 & 55174.2962 & 54924.5239 \\
\hline & $\hat{\bar{Y}}_{s p s}$ & 83190.4430 & 83153.4557 & 53369.8070 & 53221.3731 \\
\hline
\end{tabular}




\section{SUBRAMANI \& PRABAVATHY}

The percentage relative efficiencies of the proposed estimators with respect to the existing estimators were also obtained and are shown in Tables 4-5.

Table 4. Percentage Relative Efficiency of $\hat{\bar{Y}}_{S P j}$ for Population 1

\begin{tabular}{|c|c|c|c|c|c|c|c|c|c|c|}
\hline \multirow{3}{*}{$\begin{array}{l}\text { Existing } \\
\text { Estimators }\end{array}$} & \multicolumn{5}{|c|}{ For sample size $n=3$} & \multirow{2}{*}{\multicolumn{5}{|c|}{$\begin{array}{l}\text { For sample size } n=5 \\
\text { Proposed Estimators }\end{array}$}} \\
\hline & \multicolumn{5}{|c|}{ Proposed Estimators } & & & & & \\
\hline & $\hat{\bar{Y}}_{S P 1}$ & $\hat{\bar{Y}}_{S P 2}$ & $\hat{\bar{Y}}_{S P 3}$ & $\hat{\bar{Y}}_{S P 4}$ & $\hat{\bar{Y}}_{S P 5}$ & $\hat{\bar{Y}}_{S P 1}$ & $\hat{\bar{Y}}_{S P 2}$ & $\hat{\bar{Y}}_{S P 3}$ & $\hat{\bar{Y}}_{S P 4}$ & $\hat{\bar{Y}}_{S P 5}$ \\
\hline$\hat{\bar{Y}}_{r}$ & 193.86 & 195.84 & 196.19 & 192.99 & 196.36 & 167.32 & 173.57 & 171.24 & 166.18 & 171.80 \\
\hline$\hat{\bar{Y}}_{R}$ & 184.63 & 186.51 & 186.84 & 183.80 & 187.01 & 159.35 & 165.30 & 163.09 & 158.27 & 163.62 \\
\hline$\hat{\bar{Y}}_{R M 1}$ & 158.07 & 159.69 & 159.97 & 157.37 & 160.12 & 136.44 & 141.53 & 139.64 & 135.51 & 140.09 \\
\hline$\hat{\bar{Y}}_{R M 2}$ & 155.70 & 157.29 & 157.57 & 155.01 & 157.72 & 134.39 & 139.41 & 137.54 & 133.48 & 137.99 \\
\hline$\hat{\bar{Y}}_{R M 3}$ & 154.89 & 156.48 & 156.75 & 154.20 & 156.90 & 133.69 & 138.68 & 136.83 & 132.78 & 137.27 \\
\hline$\hat{\bar{Y}}_{R M 4}$ & 159.65 & 161.28 & 161.57 & 158.94 & 161.71 & 137.80 & 142.94 & 141.03 & 136.86 & 141.49 \\
\hline$\hat{\bar{Y}}_{R M 5}$ & 154.77 & 156.35 & 156.63 & 154.08 & 156.77 & 133.59 & 138.57 & 136.72 & 132.68 & 137.16 \\
\hline$\hat{\bar{Y}}_{l r}$ & 154.76 & 156.34 & 156.62 & 154.07 & 156.76 & 133.57 & 138.56 & 136.71 & 132.67 & 137.15 \\
\hline$\hat{\bar{Y}}_{M}$ & 104.88 & 105.95 & 106.14 & 104.41 & 106.24 & 106.49 & 110.47 & 108.99 & 105.77 & 109.34 \\
\hline
\end{tabular}

Table 5: Percentage Relative Efficiency of $\hat{\bar{Y}}_{S P j}$ for Population 2

\begin{tabular}{|c|c|c|c|c|c|c|c|c|c|c|}
\hline \multirow{3}{*}{$\begin{array}{l}\text { Existing } \\
\text { Estimators }\end{array}$} & \multicolumn{5}{|c|}{ For sample size $n=3$} & \multirow{2}{*}{\multicolumn{5}{|c|}{$\begin{array}{l}\text { For sample size } n=5 \\
\text { Proposed Estimators }\end{array}$}} \\
\hline & \multicolumn{5}{|c|}{ Proposed Estimators } & & & & & \\
\hline & $\hat{\bar{Y}}_{S P 1}$ & $\hat{\bar{Y}}_{S P 2}$ & $\hat{\bar{Y}}_{S P 3}$ & $\hat{\bar{Y}}_{S P 4}$ & $\hat{\bar{Y}}_{S P 5}$ & $\hat{\bar{Y}}_{S P 1}$ & $\hat{\bar{Y}}_{S P 2}$ & $\hat{\bar{Y}}_{S P 3}$ & $\hat{\bar{Y}}_{S P 4}$ & $\hat{\bar{Y}}_{S P 5}$ \\
\hline$\hat{\bar{Y}}_{r}$ & 194.13 & 195.30 & 196.40 & 193.57 & 196.45 & 167.70 & 173.71 & 171.95 & 166.94 & 172.28 \\
\hline$\hat{\bar{Y}}_{R}$ & 192.28 & 193.45 & 194.53 & 191.73 & 194.58 & 166.11 & 172.06 & 170.32 & 165.35 & 170.64 \\
\hline$\hat{\bar{Y}}_{R M 1}$ & 159.55 & 160.52 & 161.42 & 159.10 & 161.46 & 137.83 & 142.77 & 141.33 & 137.21 & 141.60 \\
\hline$\hat{\bar{Y}}_{R M 2}$ & 156.81 & 157.76 & 158.64 & 156.36 & 158.68 & 135.46 & 140.31 & 138.90 & 134.84 & 139.16 \\
\hline$\hat{\bar{Y}}_{R M 3}$ & 155.70 & 156.64 & 157.52 & 155.25 & 157.56 & 134.50 & 139.32 & 137.91 & 133.89 & 138.18 \\
\hline$\hat{\bar{Y}}_{R M 4}$ & 160.74 & 161.71 & 162.62 & 160.28 & 162.66 & 138.86 & 143.83 & 142.38 & 138.23 & 142.65 \\
\hline$\hat{\bar{Y}}_{R M 5}$ & 155.64 & 156.58 & 157.46 & 155.19 & 157.50 & 134.45 & 139.27 & 137.86 & 133.84 & 138.12 \\
\hline$\hat{\bar{Y}}_{l r}$ & 155.64 & 156.58 & 157.46 & 155.19 & 157.50 & 134.45 & 139.26 & 137.86 & 133.84 & 138.12 \\
\hline$\hat{\bar{Y}}_{M}$ & 105.03 & 105.66 & 106.26 & 104.73 & 106.28 & 106.73 & 110.56 & 109.44 & 106.25 & 109.65 \\
\hline
\end{tabular}




\section{MEDIAN BASED MODIFIED RATIO ESTIMATORS}

Tables 4 and 5 show that the percent relative efficiencies of the proposed estimators, with respect to existing estimators, range in general from 104.41 to 196.45. In particular, the PRE ranges from 166.18 to 196.45 for comparing with the SRSWOR sample mean; ranging from 158.27 to 194.58 for comparing with the ratio estimator; ranging from 132.68 to 162.66 for comparing with the modified ratio estimators; ranging from 132.67 to 157.50 for comparing with the linear regression estimator and ranging from 104.41 to 110.56 for comparing with the median based ratio estimator. This demonstrates that the proposed estimators perform better than the existing SRSWOR sample mean, ratio, modified ratio and linear regression estimators for the two populations considered. Further it is observed from the numerical comparisons that the following inequalities hold:

$$
\operatorname{MSE}\left(\hat{\bar{Y}}_{S P_{j}}\right) \leq \operatorname{MSE}\left(\hat{\bar{Y}}_{M}\right) \leq V\left(\hat{\bar{Y}}_{l r}\right) \leq \operatorname{MSE}\left(\hat{\bar{Y}}_{R M i}\right) \leq \operatorname{MSE}\left(\hat{\bar{Y}}_{R}\right) \leq V\left(\hat{\bar{Y}}_{r}\right)
$$

\section{Conclusion}

This article proposed some new median based modified ratio estimators using known quartiles and their functions of the auxiliary variable. The conditions for which the proposed estimators are more efficient than the existing estimators were derived. Further the percentage relative efficiencies of the proposed estimators with respect to existing estimators were shown to range in general from 104.41 to 196.45 for certain natural populations available in the literature. It is usually believed that the linear regression estimator is the optimum estimator for estimating the population mean whenever an auxiliary variable exists that is positively correlated with that of a study variable. However, it was shown that the proposed median based modified ratio estimators outperform not only the ratio and modified ratio estimators but also the linear regression estimator. Based on results of this study, the proposed median based modified ratio estimators are recommended for estimating finite population means.

\section{Acknowledgements}

The authors wish to express their gratitude and sincere thanks to the University Grants Commission, New Delhi for having given the financial assistance to carry out this research through the UGC-Major Research Project, and to the Editor and the referees for their effort to improve the presentation of this paper. 


\section{SUBRAMANI \& PRABAVATHY}

\section{References}

Cochran, W. G. (1977). Sampling techniques, Third Edition. Wiley Eastern Limited.

Kadilar, C., \& Cingi, H. (2004). Ratio estimators in simple random sampling. Applied Mathematics and Computation, 151: 893-902.

Kadilar, C., \& Cingi, H. (2006a). An improvement in estimating the population mean by using the correlation coefficient. Hacettepe Journal of Mathematics and Statistics, 35(1): 103-109.

Kadilar, C., \& Cingi, H. (2006b). Improvement in estimating the population mean in simple random sampling. Applied Mathematics Letters, 19: 75-79.

Kadilar, C., \& Cingi, H. (2009). Advances in Sampling Theory - Ratio Method of Estimation. Bentham Science Publishers.

Koyuncu, N., \& Kadilar, C. (2009). Efficient Estimators for the Population Mean. Hacettepe Journal of Mathematics and Statistics, 38(2): 217-225

Murthy, M. N. (1967). Sampling theory and methods. Calcutta, India: Statistical Publishing Society.

Singh, D., \& Chaudhary, F. S. (1986). Theory and analysis of sample survey designs. New Age International Publishers.

Singh, H. P., \& Kakran, M. S. (1993). A modified ratio estimator using known coefficient of kurtosis of an auxiliary character. (Unpublished Manuscript).

Singh, H. P., \& Tailor, R. (2003). Use of known correlation coefficient in estimating the finite population means. Statistics in Transition, 6(4): 555-560 .

Singh, H. P., \& Tailor, R. (2005). Estimation of finite population mean with known co-efficient of variation of an auxiliary variable. Statistica, anno $L X V(3)$ : 301-313.

Singh, G. N. (2003). On the improvement of product method of estimation in sample surveys. Journal of Indian Society of Agricultural Statistics, 56(3): 267265.

Sisodia, B. V. S., \& Dwivedi, V. K. (1981). A modified ratio estimator using co-efficient of variation of auxiliary variable. Journal of the Indian Society of Agricultural Statistics, 33(2): 13-18.

Subramani, J. (2013a). A new median based ratio estimator for estimation of the finite population mean. (Submitted for publication). 


\section{MEDIAN BASED MODIFIED RATIO ESTIMATORS}

Subramani, J. (2013b). Generalized modified ratio estimator for estimation of finite population mean. Journal of Modern Applied Statistical Methods, 12(2): 121-155.

Subramani, J., \& Kumarapandiyan, G. (2012a). Modified ratio estimators for population mean using function of quartiles of auxiliary variable. Bonfring International Journal of Industrial Engineering and Management Science, 2(2): 19-23.

Subramani, J., \& Kumarapandiyan, G. (2012b). Modified ratio estimators using known median and co-efficient of kurtosis. American Journal of Mathematics and Statistics, 2(4): 95-100.

Subramani, J., \& Kumarapandiyan, G. (2012c). Estimation of population mean using known median and co-efficient of skewness. American Journal of Mathematics and Statistics, 2(5): 101-107.

Subramani, J., \& Kumarapandiyan, G. (2013). A new modified ratio estimator of population mean when median of the auxiliary variable is known. Pakistan Journal of Statistics and Operation Research, 9(2): 137-145.

Tailor, R., \& Sharma, B. (2009). A modified ratio-cum-product estimator of finite population mean using known coefficient of variation and coefficient of kurtosis. Statistics in Transition - New Series, 10(1): 15-24.

Tin, M. (1965). Comparison of some ratio estimators. Journal of the American Statistical Association, 60: 294-307.

Yan, Z., \& Tian, B. (2010). Ratio method to the mean estimation using coefficient of skewness of auxiliary variable. ICICA 2010, Part II, CCIS 106: 103 11. 


\section{SUBRAMANI \& PRABAVATHY}

\section{Appendix A}

The derivation of the bias and the mean squared error of $\bar{Y}_{S P 1}$ are given below:

Consider

$$
\hat{\bar{Y}}_{S P 1}=\bar{y}\left(\frac{M+Q_{1}}{m+Q_{1}}\right)
$$

Let $e_{0}=\frac{\bar{y}-\bar{Y}}{\bar{Y}}$ and $e_{1}=\frac{m-M}{M}$

$\Rightarrow E\left(e_{0}\right)=0 ; E\left(e_{1}\right)=\frac{\bar{M}-M}{M}=\frac{\operatorname{Bias}(m)}{M}$

$\Rightarrow E\left(e_{0}^{2}\right)=\frac{V(\bar{y})}{\bar{Y}^{2}} ; E\left(e_{1}^{2}\right)=\frac{V(m)}{M^{2}} ; E\left(e_{0} e_{1}\right)=\frac{\operatorname{Cov}(\bar{y}, m)}{\bar{Y} M}$

The estimator $\bar{Y}_{S P 1}$ can be written in terms of $e_{0}$ and $e_{1}$ as

$$
\begin{aligned}
& \hat{\bar{Y}}_{S P 1}=\bar{Y}\left(1+e_{0}\right)\left(\frac{M+Q_{1}}{M\left(1+e_{1}\right)+Q_{1}}\right) \\
& \Rightarrow \hat{\bar{Y}}_{S P 1}=\bar{Y}\left(1+e_{0}\right)\left(\frac{M+Q_{1}}{\left(M+Q_{1}\right)+M e_{1}}\right) \\
& \Rightarrow \hat{\bar{Y}}_{S P 1}=\bar{Y}\left(1+e_{0}\right)\left(\frac{1}{1+\left(\frac{M}{M+Q_{1}}\right) e_{1}}\right) \\
& \Rightarrow \hat{\bar{Y}}_{S P 1}=\bar{Y}\left(1+e_{0}\right)\left(\frac{1}{1+\theta_{1}^{\prime} e_{1}}\right) ; \text { where } \theta_{1}^{\prime}=\frac{M}{M+Q_{1}}
\end{aligned}
$$




\section{MEDIAN BASED MODIFIED RATIO ESTIMATORS}

$\Rightarrow \hat{\bar{Y}}_{S P 1}=\bar{Y}\left(1+e_{0}\right)\left(1+\theta_{1}^{\prime} e_{1}\right)^{-1}$

Neglecting the terms of higher order, we have

$$
\begin{aligned}
& \hat{\bar{Y}}_{S P 1}=\bar{Y}\left(1+e_{0}\right)\left(1-\theta_{1}^{\prime} e_{1}+\theta_{1}^{\prime 2} e_{1}^{2}\right) \\
& \Rightarrow \hat{\bar{Y}}_{S P 1}=\bar{Y}+\bar{Y} e_{0}-\bar{Y} \theta_{1}^{\prime} e_{1}-\bar{Y} \theta_{1}^{\prime} e_{0} e_{1}+\bar{Y} \theta_{1}^{\prime 2} e_{1}^{2} \\
& \Rightarrow \hat{\bar{Y}}_{S P 1}-\bar{Y}=\bar{Y} e_{0}-\bar{Y} \theta_{1}^{\prime} e_{1}-\bar{Y} \theta_{1}^{\prime} e_{0} e_{1}+\bar{Y} \theta_{1}^{\prime 2} e_{1}^{2}
\end{aligned}
$$

Taking expectations on both sides of (A4) we have,

$$
\begin{aligned}
& E\left(\hat{\bar{Y}}_{S P 1}-\bar{Y}\right)=\bar{Y} E\left(e_{0}\right)-\bar{Y} \theta_{1}^{\prime} E\left(e_{1}\right)-\bar{Y} \theta_{1}^{\prime} E\left(e_{0} e_{1}\right)+\bar{Y} \theta_{1}^{\prime 2} E\left(e_{1}^{2}\right) \\
& \Rightarrow E\left(\hat{\bar{Y}}_{S P 1}-\bar{Y}\right)=\bar{Y}\left\{\theta_{1}^{\prime 2} C_{m m}^{\prime}-\theta_{1}^{\prime} C_{y m}^{\prime}-\theta_{1}^{\prime} \frac{\operatorname{Bias}(m)}{M}\right\} \text { from (A2) and (A3) } \\
& \Rightarrow \operatorname{Bias}\left(\hat{\bar{Y}}_{S P 1}\right)=\bar{Y}\left\{\theta_{1}^{\prime 2} C_{m m}^{\prime}-\theta_{1}^{\prime} C_{y m}^{\prime}-\theta_{1}^{\prime} \frac{\operatorname{Bias}(m)}{M}\right\}
\end{aligned}
$$

The derivation of mean squared error of $\bar{Y}_{S P 1}$ is given below:

$$
\begin{aligned}
& \operatorname{MSE}\left(\hat{\bar{Y}}_{S P 1}\right)=E\left(\hat{\bar{Y}}_{S P 1}-\bar{Y}\right)^{2}=E\left(\bar{Y} e_{0}-\bar{Y} \theta_{1}^{\prime} e_{1}\right)^{2} \\
& \Rightarrow \operatorname{MSE}\left(\hat{\bar{Y}}_{S P 1}\right)=\bar{Y}^{2}\left\{E\left(e_{0}^{2}\right)+\theta_{1}^{\prime 2} E\left(e_{1}^{2}\right)-2 \theta_{1}^{\prime} E\left(e_{0} e_{1}\right)\right\} \\
& \Rightarrow \operatorname{MSE}\left(\hat{\bar{Y}}_{S P 1}\right)=\bar{Y}^{2}\left\{\frac{V(\bar{y})}{\bar{Y}^{2}}+\theta_{1}^{\prime 2} \frac{V(m)}{M^{2}}-2 \theta_{1}^{\prime} \frac{\operatorname{Cov}(\bar{y}, m)}{\bar{Y} M}\right\} \\
& \Rightarrow \operatorname{MSE}\left(\hat{\bar{Y}}_{S P 1}\right)=V(\bar{y})+\frac{\bar{Y}^{2}}{M^{2}} \theta_{1}^{\prime 2} V(m)-2 \frac{\bar{Y}}{M} \theta_{1}^{\prime} \operatorname{Cov}(\bar{y}, m)
\end{aligned}
$$




\section{SUBRAMANI \& PRABAVATHY}

$\Rightarrow \operatorname{MSE}\left(\hat{\bar{Y}}_{S P 1}\right)=V(\bar{y})+R^{\prime 2} \theta_{1}^{\prime 2} V(m)-2 R^{\prime} \theta_{1}^{\prime} \operatorname{Cov}(\bar{y}, m) ; R^{\prime}=\frac{\bar{Y}}{M}$

In the Similar manner, the bias and mean squared error of $\hat{\bar{Y}}_{S P 2}, \hat{\bar{Y}}_{S P 3}, \hat{\bar{Y}}_{S P 4}$ and $\hat{\bar{Y}}_{S P 5}$ can be obtained.

\section{Appendix B}

The conditions for which the proposed estimators perform better than the existing estimators are derived here and are given below:

\section{Comparison with that of SRSWOR sample mean}

$$
\begin{aligned}
& \text { Consider } \operatorname{MSE}\left(\hat{\bar{Y}}_{S P_{j}}\right) \leq V\left(\hat{\bar{Y}}_{r}\right) \\
& \Rightarrow V(\bar{y})+R^{\prime 2} \theta_{j}^{\prime 2} V(m)-2 R^{\prime} \theta_{j}^{\prime} \operatorname{Cov}(\bar{y}, m) \leq V(\bar{y}) \\
& \Rightarrow R^{\prime 2} \theta_{j}^{\prime 2} V(m)-2 R^{\prime} \theta_{j}^{\prime} \operatorname{Cov}(\bar{y}, m) \leq 0 \\
& \Rightarrow R^{\prime 2} \theta_{j}^{\prime 2} V(m) \leq 2 R^{\prime} \theta_{j}^{\prime} \operatorname{Cov}(\bar{y}, m) \\
& \Rightarrow \operatorname{Cov}(\bar{y}, m) \geq \frac{R^{\prime} \theta_{j}^{\prime} V(m)}{2}, j=1,2,3,4,5 \\
& \Rightarrow \operatorname{Cov}(\bar{y}, m) \geq \frac{\bar{Y} M \theta_{j}^{\prime} C_{m m}^{\prime}}{2} \\
& \Rightarrow 2 C_{y m}^{\prime} \geq \theta_{j}^{\prime} C_{m m}^{\prime}, j=1,2,3,4,5
\end{aligned}
$$




\section{MEDIAN BASED MODIFIED RATIO ESTIMATORS}

\section{Comparison with that of Ratio Estimator}

$$
\begin{aligned}
& \text { Consider } \operatorname{MSE}\left(\hat{\bar{Y}}_{S P_{j}}\right) \leq \operatorname{MSE}\left(\hat{\bar{Y}}_{R}\right) \\
& \Rightarrow V(\bar{y})+R^{\prime 2} \theta_{j}^{\prime 2} V(m)-2 R^{\prime} \theta_{j}^{\prime} \operatorname{Cov}(\bar{y}, m) \leq V(\bar{y})+R^{2} V(\bar{x})-2 R \operatorname{Cov}(\bar{y}, \bar{x}) \\
& \Rightarrow R^{\prime 2} \theta_{j}^{\prime 2} V(m)-2 R^{\prime} \theta_{j}^{\prime} \operatorname{Cov}(\bar{y}, m) \leq R^{2} V(\bar{x})-2 \operatorname{RCov}(\bar{y}, \bar{x}) \\
& \Rightarrow R^{\prime 2} \theta_{j}^{\prime 2} V(m)-R^{2} V(\bar{x}) \leq 2 R^{\prime} \theta_{j}^{\prime} \operatorname{Cov}(\bar{y}, m)-2 \operatorname{RCov}(\bar{y}, \bar{x}) \\
& \Rightarrow \frac{\bar{Y}^{2}}{M^{2}} \theta_{j}^{\prime 2} V(m)-\frac{\bar{Y}^{2}}{\bar{X}^{2}} V(\bar{x}) \leq 2 \frac{\bar{Y}}{M} \theta_{j}^{\prime} \operatorname{Cov}(\bar{y}, m)-2 \frac{\bar{Y}}{\bar{X}} \operatorname{Cov}(\bar{y}, \bar{x}) \\
& \Rightarrow \theta_{j}^{\prime 2} \frac{V(m)}{M^{2}}-\frac{V(\bar{x})}{\bar{X}^{2}} \leq 2\left\{\theta_{j}^{\prime} \frac{\operatorname{Cov}(\bar{y}, m)}{\bar{Y} M}-\frac{\operatorname{Cov}(\bar{y}, \bar{x})}{\overline{Y X}}\right\} \\
& \Rightarrow \theta_{j}^{\prime 2} C_{m m}^{\prime}-C_{x x}^{\prime} \leq 2\left\{\theta_{j}^{\prime} C_{y m}^{\prime}-C_{y x}^{\prime}\right\} ; j=1,2,3,4,5
\end{aligned}
$$

\section{Comparison with that of Modified Ratio Estimators}

Consider $\operatorname{MSE}\left(\hat{\bar{Y}}_{S P j}\right) \leq \operatorname{MSE}\left(\hat{\bar{Y}}_{R M i}\right)$

$$
\begin{aligned}
& \Rightarrow V(\bar{y})+R^{\prime 2} \theta_{j}^{\prime 2} V(m)-2 R^{\prime} \theta_{j}^{\prime} \operatorname{Cov}(\bar{y}, m) \leq V(\bar{y})+R^{2} \theta_{i}^{2} V(\bar{x})-2 R \theta_{i} \operatorname{Cov}(\bar{y}, \bar{x}) \\
& \Rightarrow R^{\prime 2} \theta_{j}^{\prime 2} V(m)-2 R^{\prime} \theta_{j}^{\prime} \operatorname{Cov}(\bar{y}, m) \leq R^{2} \theta_{i}^{2} V(\bar{x})-2 R \theta_{i} \operatorname{Cov}(\bar{y}, \bar{x}) \\
& \Rightarrow R^{\prime 2} \theta_{j}^{\prime 2} V(m)-R^{2} \theta_{i}^{2} V(\bar{x}) \leq 2 R^{\prime} \theta_{j}^{\prime} \operatorname{Cov}(\bar{y}, m)-2 R \theta_{i} \operatorname{Cov}(\bar{y}, \bar{x}) \\
& \Rightarrow \frac{\bar{Y}^{2}}{M^{2}} \theta_{j}^{\prime 2} V(m)-\frac{\bar{Y}^{2}}{\bar{X}^{2}} \theta_{i}^{2} V(\bar{x}) \leq 2 \frac{\bar{Y}}{M} \theta_{j}^{\prime} \operatorname{Cov}(\bar{y}, m)-2 \frac{\bar{Y}}{\bar{X}} \theta_{i} \operatorname{Cov}(\bar{y}, \bar{x})
\end{aligned}
$$




\section{SUBRAMANI \& PRABAVATHY}

$$
\begin{aligned}
& \Rightarrow \theta_{j}^{\prime 2} \frac{V(m)}{M^{2}}-\theta_{i}^{2} \frac{V(\bar{x})}{\bar{X}^{2}} \leq 2\left\{\theta_{j}^{\prime} \frac{\operatorname{Cov}(\bar{y}, m)}{\bar{Y} M}-\theta_{i} \frac{\operatorname{Cov}(\bar{y}, \bar{x})}{\bar{Y} \bar{X}}\right\} \\
& \Rightarrow \theta_{j}^{\prime 2} C_{m m}^{\prime}-\theta_{i}^{2} C_{x x}^{\prime} \leq 2\left\{\theta_{j}^{\prime} C_{y m}^{\prime}-\theta_{i} C_{y x}^{\prime}\right\} ; i, j=1,2,3,4,5
\end{aligned}
$$

\section{Comparison with that of Linear Regression Estimator}

$$
\begin{aligned}
& \text { Consider } \operatorname{MSE}\left(\hat{\bar{Y}}_{S P_{j}}\right) \leq V\left(\hat{\bar{Y}}_{l r}\right) \\
& \Rightarrow V(\bar{y})+R^{\prime 2} \theta_{j}^{\prime 2} V(m)-2 R^{\prime} \theta_{j}^{\prime} \operatorname{Cov}(\bar{y}, m) \leq V(\bar{y})\left(1-\rho^{2}\right) \\
& \Rightarrow R^{\prime 2} \theta_{j}^{\prime 2} V(m)-2 R^{\prime} \theta_{j}^{\prime} \operatorname{Cov}(\bar{y}, m) \leq-V(\bar{y})\left(\frac{[\operatorname{Cov}(\bar{y}, \bar{x})]^{2}}{V(\bar{x}) * V(\bar{y})}\right) \\
& \Rightarrow 2 R^{\prime} \theta_{j}^{\prime} \operatorname{Cov}(\bar{y}, m)-R^{\prime 2} \theta_{j}^{\prime 2} V(m) \geq \frac{[\operatorname{Cov}(\bar{y}, \bar{x})]^{2}}{V(\bar{x})} ; j=1,2,3,4,5 \\
& \Rightarrow 2 \frac{\bar{Y}}{M} \theta_{j}^{\prime} \operatorname{Cov}(\bar{y}, m)-\frac{\bar{Y}^{2}}{M^{2}} \theta_{j}^{\prime 2} V(m) \geq \frac{[\operatorname{Cov}(\bar{y}, \bar{x})]^{2}}{V(\bar{x})} \\
& \Rightarrow 2 \bar{Y}^{2} \theta_{j}^{\prime} C_{y m}^{\prime}-\bar{Y}^{2} \theta_{j}^{\prime 2} C_{m m}^{\prime} \geq \frac{[\operatorname{Cov}(\bar{y}, \bar{x})]^{2}}{V(\bar{x})} \\
& \Rightarrow 2 \theta_{j}^{\prime} C_{y m}^{\prime}-\theta_{j}^{\prime 2} C_{m m}^{\prime} \geq \frac{\left[C_{y x}^{\prime}\right]^{2}}{C_{x x}^{\prime}}, j=1,2,3,4,5
\end{aligned}
$$




\section{MEDIAN BASED MODIFIED RATIO ESTIMATORS}

\section{Comparison with that of Median Based Ratio Estimator}

$$
\begin{aligned}
& \text { Consider } \operatorname{MSE}\left(\hat{\bar{Y}}_{S P j}\right) \leq \operatorname{MSE}\left(\hat{\bar{Y}}_{M}\right) \\
& \Rightarrow V(\bar{y})+R^{\prime 2} \theta_{j}^{\prime 2} V(m)-2 R^{\prime} \theta_{j}^{\prime} \operatorname{Cov}(\bar{y}, m) \leq V(\bar{y})+R^{\prime 2} V(m)-2 R^{\prime} \operatorname{Cov}(\bar{y}, m) \\
& \Rightarrow R^{\prime 2} \theta_{j}^{\prime 2} V(m)-2 R^{\prime} \theta_{j}^{\prime} \operatorname{Cov}(\bar{y}, m) \leq R^{\prime 2} V(m)-2 R^{\prime} \operatorname{Cov}(\bar{y}, m) \\
& \Rightarrow R^{\prime 2} \theta_{j}^{\prime 2} V(m)-R^{\prime 2} V(m) \leq 2 R^{\prime} \theta_{j}^{\prime} \operatorname{Cov}(\bar{y}, m)-2 R^{\prime} \operatorname{Cov}(\bar{y}, m) \\
& \Rightarrow R^{\prime} V(m)\left(\theta_{j}^{\prime 2}-1\right) \leq 2\left(\theta_{j}^{\prime}-1\right) \operatorname{Cov}(\bar{y}, m) \\
& \Rightarrow R^{\prime} V(m)\left(\theta_{j}^{\prime}-1\right)\left(\theta_{j}^{\prime}+1\right) \leq 2\left(\theta_{j}^{\prime}-1\right) \operatorname{Cov}(\bar{y}, m) \\
& \Rightarrow \operatorname{Cov}(\bar{y}, m) \leq \frac{R^{\prime}\left(\theta_{j}^{\prime}+1\right) V(m)}{2} \text { Since } \theta_{j}^{\prime}<1 ; j=1,2,3,4,5 \\
& \Rightarrow \operatorname{Cov}(\bar{y}, m) \leq \frac{\bar{Y} M\left(\theta_{j}^{\prime}+1\right) C_{m m}^{\prime}}{2} \text { Since } \theta_{j}^{\prime}<1 \\
& \Rightarrow 2 C_{y m}^{\prime} \leq\left(\theta_{j}^{\prime}+1\right) C_{m m}^{\prime}, j=1,2,3,4,5
\end{aligned}
$$

